\title{
COMPLEJIDAD VIVENCIAL Y EMOCIONES EN LA FORMACIÓN DOCENTE: Casos ilustrativos con estudiantes de práctica profesional y profesorado principiante
}

\author{
Mariela de Lourdes Urbina-Cerda \\ mariela.urbina@umce.cl \\ https://orcid.org/0000-0001-9391-1564 \\ Universidad Metropolitana de Ciencias de la Educación (UMCE) \\ Santiago, Chile. \\ Jorge Iván Ávila-Contreras \\ jorge.avila@umce.cl \\ http://orcid.org/0000-0002-6045-1319
}

Universidad Metropolitana de Ciencias de la Educación (UMCE)

Santiago, Chile.

Recibido: 02/01/2021 Aceptado: 09/02/2021

\begin{abstract}
Resumen
En este trabajo, con base en la noción de complejidad vivencial y considerando las características de este nuevo siglo en que ya estamos inmersos, se reflexionan limitaciones de los estudios que atienden a la mejora de la calidad en la formación docente. Se ilustran evidencias de complejidades vivenciales y emociones provenientes de estudiantes de pedagogía en matemática que cursan su práctica profesional $\mathrm{y}$, también, de profesorado principiante de educación primaria. Los estudiantes en práctica profesional se debaten entre lo recibido como formación y el encuentro con la realidad del contexto educativo, se observa un entretejido de emociones diferentes asociadas a la gestión del aula que confluyen (cobran forma) en conexión con su buena relación y cercanía con sus estudiantes. Por su parte, entre las experiencias del profesorado principiante se tiene la valoración de su rol docente, el vivenciar(se) en una experiencia solitaria y quijotesca; $y$, la formación de sus estudiantes problematizando la díada formación integral de una persona versus el rendimiento.
\end{abstract}

Palabras clave: Complejidad vivencial. Emociones y formación docente. Emociones y profesorado principiante. Calidad en la formación docente.

\section{VIVENDO A COMPLEXIDADE E EMOÇÕES NO TREINAMENTO DE ENSINO:}

\section{Casos ilustrativos com alunos de prática profissional e professores iniciantes}

\begin{abstract}
Resumo
Neste trabalho, com base na noção de complexidade experiencial e considerando as características deste novo século em que já estamos imersos, refletem-se as limitações dos estudos que abordam a melhoria da qualidade da formação docente. São ilustradas evidências de complexidades experienciais e emoções de alunos de pedagogia matemática que estão em sua prática profissional e também de professores iniciantes do ensino fundamental. Os alunos em prática profissional debatem entre o que receberam como formação e o encontro com a realidade do contexto educacional, observa-se um entrelaçamento de diferentes emoções associadas à gestão da sala de aula que convergem (ganham forma) no seu bom relacionamento
\end{abstract}


e proximidade com o vosso. alunos. Por outro lado, entre as vivências das professoras iniciantes está a avaliação de seu papel docente, a vivência (si) em uma experiência solitária e quixotesca; e, a formação de seus alunos problematizando a díade formação integral da pessoa versus performance.

Palavras chave: Complexidade experiencial. Emoções e formação de professores. Professores iniciantes e emoções. Qualidade na formação de professores.

\title{
EXPERIENCE COMPLEXITY AND EMOTIONS IN TEACHING TRAINING: Illustrative cases with professional practice students and beginning teachers
}

\begin{abstract}
In this work, based on the notion of experiential complexity and considering the characteristics of this new century in which we are already immersed, limitations of the studies that address the improvement of quality in teacher training are reflected. Evidence of experiential complexities and emotions from mathematics pedagogy students who are in their professional practice and also from beginning teachers of primary education are illustrated. Students in professional practice debate between what they have received as training and the encounter with the reality of the educational context, an interweaving of different emotions associated with classroom management is observed that converge (take shape) in connection with their good relationship and closeness with your students. On the other hand, among the experiences of the beginning teachers there is the evaluation of their teaching role, the experience (oneself) in a solitary and quixotic experience; and, the formation of its students problematizing the dyad integral formation of a person versus performance.
\end{abstract}

Keywords: Experiential complexity. Emotions and teacher training. Beginning teachers and emotions. Quality in teacher training.

\section{Introducción}

Los procesos de formación docente se entrecruzan con múltiples escenarios sociales y culturales que en las últimas décadas han cambiado drásticamente. Durante el siglo XX se transitó desde una sociedad industrializada hacia una con foco en la información, las comunicaciones y las tecnologías. Hoy en día, en el cotidiano y lo educativo, se evidencia fuertemente el uso de las tecnologías (Cózar-Gutiérrez, Moya-Martínez, Hernández-Bravo y Hernández-Bravo, 2016) lo que trae consigo un dinamismo e inmediatez para desenvolverse en los procesos educativos, diferente al de décadas pasadas. De acuerdo a Casillas y Ramírez (2019) la época contemporánea se distingue por el incesante desarrollo de la revolución tecnológica - fundada en la digitalización y el uso extendido de las computadoras y el acceso a Internet-que resulta en la conformación de una cultura digital como nuevo referente simbólico. 
En los últimos decenios, además, se han valorado nuevas facetas de complejidad intrínsecas a lo humano, generando un deslizamiento en el modo de concebir la naturaleza humana (Morín, 1990; Najmanovich, 2001; Varela, 1990, 2000). En estas aproximaciones, se plantea que el acto de conocer del humano acontece con y desde todo su ser, emocional, animal y racional, no se conoce únicamente a partir de la dimensión racional.

Con el paradigma de la complejidad, Morín (1990) realza al sujeto como homo complexus en lugar de exclusivamente como un homo sapiens, pone al centro la naturaleza imbricada del humano mediante bucles indisociables de su ser, que consideran su dimensión animal, cultural, planetaria, impulsiva, entre otras, como un entretejido complejo. Najmanovich (2001), en lugar del cuerpo de la modernidad como un cuerpo material, objetivo e independiente, refiere a un cuerpo cognitivo emocional como un sujeto encarnado. Varela (1990, 2000), desde sus investigaciones en las ciencias cognitivas y las neurociencias, sostiene que la mente humana es un fenómeno indisociable del cuerpo y propone con su perspectiva de la enacción, que el conocer se da en el sujeto a partir de una acción efectiva en el mundo.

En el ámbito educativo, se observa también una ampliación de los aspectos a tener en cuenta para la formación docente, entre ellos, la dimensión emocional. Antaño, las emociones eran consideradas como un factor que correspondía exclusivamente al ámbito personal, sin embargo, actualmente una amplia literatura la sitúa como un elemento fundamental para la formación docente (Bächler, Meza, Mendoza y Poblete, 2020; Zembylas, Watkins, Becerra y Aleu, 2019; Ávila, 2018a, 2018b; Casassus, 2015; Day, 2011; Gómez-Chacón, 2010; OCDE/CERI, 2009; Badia, 2014; Hargreaves, 2005, 2001; entre otros).

En particular, en este artículo, para el estudio de las emociones se usa la perspectiva de la complejidad vivencial (Ávila, 2018a), más que una postura asociativa que vincule emociones positivas o negativas con situaciones de diversa índole, en esta aproximación interesa explorar aspectos vivenciales que intervienen en la ocurrencia de una emoción. Así, se busca visibilizar con mayor profundidad fenómenos complejos imbricados en la formación docente.

En una primera parte del escrito, se problematizan informes internacionales vinculados a la calidad educativa (TALIS, SITEAL, TERCE y PISA) reflexionando acerca de la necesidad de incorporar facetas relacionadas a la complejidad humana, acorde a este siglo XXI, tomando en cuenta entre otras a la dimensión vivencial y emocional de los sujetos implicados en el proceso educativo. En segundo lugar, se presentan algunas ideas claves del pensamiento 
complejo y la noción de complejidad vivencial. Posteriormente, se ilustra lo expuesto con resultados de un estudio que explora con esta perspectiva las emociones de estudiantes de pedagogía en matemáticas en el contexto de su práctica profesional y, también, de una investigación en curso que aborda, entre otras temáticas, la inserción profesional docente de profesorado principiante de educación primaria.

\section{Informes sobre la calidad educativa: limitaciones desde una mirada con foco en la multidimensionalidad y complejidad humana}

Informes internacionales como TALIS, SITEAL, TERCE y PISA buscan medir e identificar el impacto que tienen las políticas públicas en la mejora de la calidad de la educación, sin embargo, pensamos que se profundiza poco en aquellas complejidades multidimensionales y factores que permiten la construcción de una identidad profesional docente más fortalecida, de manera de incidir más fuertemente en la forma en que el profesorado vivencia su devenir profesional y, por ende, la forma en cómo se aprende y enseña en los procesos educativos.

En general al evaluar la profesión docente, estos informes invisibilizan factores complejos intrínsecos a las prácticas docentes y, con ello, se sigue llenando una especie de mochila emocional que carga el profesorado, haciéndoles reiteradamente replantearse el compromiso contraído con la educación.

A continuación, se exponen de manera más específica algunas limitaciones que -con base en este posicionamiento epistemológico- identificamos en cuatro documentos representativos de las directrices en el ámbito de las políticas educativas, a saber, los informes: TALIS, SITEAL, TERCE y PISA.

\section{TALIS}

El informe TALIS 2018 (OECD, 2019) coloca el foco en la gestión del aula, los procesos que se dan en los centros educativos y la preparación tanto en la formación inicial docente como en la formación continua del profesorado (Ver Cuadro 1)

Cuadro 1.

Síntesis de la Guía del profesorado TALIS 2018.

\begin{tabular}{|l|ll|}
\hline \multicolumn{1}{|c|}{ Dimensiones } & \multicolumn{1}{c|}{ Elementos } \\
\hline $\begin{array}{l}\text { ¿Qué está pasando en } \\
\text { el aula? }\end{array}$ & $\bullet$ & Formación para la gestión del aula: autoeficacia de los profesores \\
\hline
\end{tabular}




\begin{tabular}{|l|ll|}
\hline & $\begin{array}{l}\text { Apoyando a los docentes noveles: prioridades de gasto para la } \\
\text { educación secundaria obligatoria }\end{array}$ \\
\hline $\begin{array}{l}\text { ¿Qué está pasando en } \\
\text { el centro educativo? }\end{array}$ & $\bullet \begin{array}{l}\text { Apoyando la enseñanza para la diversidad } \\
\bullet\end{array}$ & Paciendo el tiempo para el liderazgo instruccional \\
\hline $\begin{array}{l}\text { ¿Cómo se preparan } \\
\text { los profesores y los } \\
\text { líderes escolares para } \\
\text { su trabajo? }\end{array}$ & $\bullet$ & - $\begin{array}{l}\text { Apreparando a los docentes } \\
\text { Apoyando a los profesores nuevos en sus escuelas }\end{array}$ \\
\hline $\begin{array}{l}\text { ¿Cómo se mantienen } \\
\text { actualizados para el } \\
\text { desempeño de su } \\
\text { profesión los } \\
\text { profesores y los } \\
\text { líderes escolares? }\end{array}$ & $\bullet \quad$ Formando a líderes escolares \\
\hline
\end{tabular}

Fuente: Elaborado por los autores

Una de las limitaciones encontradas al analizar este documento es la definición -o falta de ella- acerca de lo que se entiende por Gestión de aula. Según Pardo (2018), esta es:

Un conjunto de acciones de variada índole que permiten construir un contexto propicio para el aprendizaje. Estas acciones van desde la organización de los contenidos y el diseño instruccional propiamente tal, hasta la promoción de interacciones pacíficas y colaborativas entre los estudiantes. (p. 1)

En contraste con lo declarado en el Cuadro 1, llama la atención que las respuestas presentadas en el informe, en relación al profesorado de secundaria de los países OCDE participantes en el estudio, reducen la gestión de aula sólo a lo normativo conductual sin considerar otros factores que la conforman. Pardo (2018, citando a Darling-Hammond \& Bransford, 2005, p. 2) establece que los(as) profesores(as) gestionan el aula cuando:

- Organizan y estructuran su clase.

- Crean un currículum significativo y entusiasman a los estudiantes a aprender, despertando la motivación intrínseca.

- Promueven comunidades de aprendizaje.

- Reparan y restituyen el comportamiento respetuosamente.

- Propician el desarrollo moral. 
De acuerdo a lo anterior, la Gestión de aula implica una visión integral e integrada de diversos factores pedagógicos, didácticos, de configuración emocional, social y ética que trascienden lo meramente normativo, es decir, gestionar el aula es más que enfocarse en la conducta disruptiva o normativa de la clase. Podemos decir que es una clave de SOL (sujetos, objetos, lugares) que orienta la acción docente considerando a sí mismo(a) y a otros(as), a los objetos que los configuran (biografía personal y académica, vivencias, emociones, acciones que conforman sus constructos, etc.) y a la estructura del mundo que les da sentido, los lugares entendidos como la diversidad de contextos físicos, psicológicos, históricos, biológicos y socioculturales en los cuales se construyen sujetos y objetos en una danza que imbrica la multidimensionalidad del ser humano.

Consideramos que no es posible considerar la enseñanza, participación del estudiantado y actividades mejoradas como elementos distintos de la Gestión del aula. Siguiendo a Pardo (2018, citando a Darling-Hammond \& Bransford, 2005) estos elementos son parte de los distintos componentes que la conforman.

Asimismo, de acuerdo a este informe las y los docentes de secundaria, participantes en el estudio, externalizan a factores materiales su función y profesionalización del aula, es decir, los factores humanos, emocionales y profesionales que impactan en los procesos de enseñanza y aprendizaje y, por ende, en sus estudiantes, no son considerados ni visualizados como variables a desarrollar o necesarios para la mejora docente en sus centros educativos cuando se les pide considerar recursos adicionales.

Según TALIS 2018, desde el punto de vista de los centros educativos, se espera que el profesorado "esté preparado para tratar la diversidad en todas sus formas en el aula" (p. 9) y que "los líderes escolares pueden diseñar políticas y aplicar prácticas que aseguren que todos los alumnos, independientemente de su origen socioeconómico y cultural, puedan aprender" (p.10); sin embargo, no se establece cómo estos líderes escolares podrían gestionar política y administrativamente las responsabilidades que les competen a los centros educativos en estas acciones. Nuevamente recae sobre el profesorado una acción quijotesca y solitaria sin que las estructuras directivas de las instituciones escolares asuman la responsabilidad en la gestión educativa de la escuela.

Otro aspecto que llama la atención es la idea de establecer tiempos para el liderazgo instruccional desconociendo la realidad en tiempo de destinación a las clases lectivas y recursos 
financieros que requiere esa visión. En Chile, por ejemplo, las escuelas públicas y particulares subvencionadas permiten muy pocas horas no lectivas al profesorado haciendo imposible que puedan "aprovechar cualquier oportunidad para desarrollar sus capacidades de liderazgo instruccional, participando en actividades de desarrollo profesional, colaborando e interactuando en eventos con otros directores escolares, así como observando, realizando mentorías y ofreciendo retroalimentación a los profesores" (p. 13).

Se propone que "las autoridades educativas o líderes escolares a cargo de los recursos humanos deben asegurarse de que sus docentes tengan la formación suficiente en el contenido de sus asignaturas, pedagogía y prácticas docentes en el aula” (p. 17). Sin embargo, esta visión no considera las características necesarias, tanto para el profesorado como para sus estudiantes, que permitan dar respuestas a las necesidades del nuevo milenio, por ejemplo, la complejidad humana, la dimensión afectiva y emocional, la formación ciudadana, la responsabilidad social e individual, la alimentación saludable y autosustentable, entre otras, que han quedado patentes sobre todo en el actual contexto de pandemia COVID 19 que azota a la humanidad.

\section{SITEAL}

El informe SITEAL (2014) sobre tendencias sociales y educativas en América Latina, se estructura en cinco capítulos: Subjetividades juveniles de la tecnocultura digital; Políticas de integración de TIC en América Latina; Políticas de infraestructura, equipamiento y conectividad; Políticas de integración TIC en las instituciones educativas; y, TIC y formación docente: formación inicial y desarrollo profesional docente.

Este informe, en el 2014, ya colocaba el foco en la necesidad de integrar las tecnologías en la educación y hacía hincapié en la desigualdad del acceso a ellas por parte de toda la población, pues se consideraba que la inclusión digital implicaba justicia social. El estudio consideraba que las nuevas generaciones habían nacido en un mundo tecnológico y, por tanto, estaban capacitadas para utilizar la tecnología. Sin embargo, la realidad frente al azote del COVID-19, donde países como Chile tuvieron que incorporar las clases online en todos sus niveles educativos, dejó de manifiesto que ni las nuevas generaciones ni el profesorado estaban capacitados para usar la tecnología. Además, en este contexto de pandemia, diversas reflexiones en el ámbito educativo centraron la atención en la importancia del acceso igualitario a la 
tecnología y en la superación de las brechas de desigualdad evidenciadas a partir de ella (Ordorika, 2020; CEPAL-UNESCO, 2020; Tarabini, 2020).

Somos consumidores de tecnología, pero ello no implica que estemos capacitados para su uso, que podamos comprender y evaluar el impacto de la tecnología - o la falta de ella- en nuestro quehacer cotidiano o que tengamos un acceso igualitario a ella ¿somos capaces de reflexionar y/o usar conscientemente la información que se desprende del uso de las tecnologías? ¿cómo impacta esta desigualdad de acceso a la información en el desarrollo de las personas? ¿como profesorado desarrollamos metodologías que permitan la igualdad de condiciones para superar la brecha digital en el estudiantado más vulnerable?

Estamos en presencia de una cultura digital para la cual el profesorado no ha sido formado y las políticas analizadas en este informe dan cuenta de la necesidad de elementos y metodologías que incorporen las TIC, pero no hace alusión a cómo se enseña a analizar la información, a distinguir la información correcta de la incorrecta, cómo se aprende a utilizar las TIC más allá de su consumo sino como parte fundamental de una nueva era y nuevas culturas que interactúan en los diversos espacios digitales y/o sociales, cómo somos parte de la transformación social que permita superar las brechas de desigualdad social y digital desde la complejidad vivencial y multidimensional que nos constituye.

\section{TERCE}

El Informe de resultados: logros de aprendizaje corresponde al Tercer estudio regional comparativo y explicativo TERCE 2015 (UNESCO, 2015) y considera 15 países evaluados de América Latina en relación con la lectura, escritura, matemáticas y ciencias naturales (UNESCO tiene publicado en su sitio web que el cuarto informe comparativo, ERCE 2019, estará disponible durante el año 2021). Los resultados corresponden al enfoque de la prueba aplicada en cada una de las cuatro materias señaladas y en los dominios y procesos asociadas a ellas. Asimismo, destaca el rendimiento y desempeño académico en dichas pruebas de estudiantes de tercer y sexto grado.

Cuadro 2.

Síntesis estructura Informe TERCE 2015

\begin{tabular}{|l|l|l|}
\hline $\begin{array}{c}\text { Evaluación de } \\
\text { logros de: }\end{array}$ & Ejes temáticos & Niveles cognitivos \\
\hline $\begin{array}{l}\text { Aprendizaje en } \\
\text { lectura }\end{array}$ & $\bullet$ Comprensión de textos & $\begin{array}{l}\text { 1. Comprensión literal. } \\
\text { 2. Comprensión inferencial. }\end{array}$ \\
\hline
\end{tabular}




\begin{tabular}{|c|c|c|}
\hline & - Metalingüístico y teórico. & 3. Comprensión crítica. \\
\hline $\begin{array}{l}\text { Aprendizaje en } \\
\text { Matemática }\end{array}$ & $\begin{array}{ll}\text { - } & \text { Dominio numérico } \\
\text { - } & \text { Dominio Geométrico } \\
\text { - Dominio de la medición } \\
\text { - Dominio estadístico } \\
\text { - Dominio de la variación }\end{array}$ & $\begin{array}{l}\text { 1. } \begin{array}{l}\text { Reconocimiento de } \\
\text { objetos y elementos }\end{array} \\
\text { 2. } \begin{array}{l}\text { Solución de problemas } \\
\text { simples }\end{array} \\
\text { 3. } \\
\begin{array}{l}\text { Solución de problemas } \\
\text { complejos }\end{array}\end{array}$ \\
\hline $\begin{array}{l}\text { Aprendizaje en } \\
\text { Ciencias } \\
\text { Naturales }\end{array}$ & $\begin{array}{ll}\text { - } & \text { Salud } \\
\text { - } & \text { Seres vivos } \\
\text { - } & \text { Ambiente } \\
\text { - } & \text { La Tierra y el sistema Solar } \\
\text { - } & \text { Materia y energia }\end{array}$ & $\begin{array}{l}\text { 1. } \text { Reconocimiento de } \\
\text { información y conceptos } \\
\text { 2. } \text { Comprensión y } \\
\text { aplicación de los mismos } \\
\text { 3. El pensamiento científico } \\
\text { y la resolución de } \\
\text { problemas }\end{array}$ \\
\hline $\begin{array}{l}\text { Aprendizaje en } \\
\text { Escritura }\end{array}$ & $\begin{array}{ll}\text { - } & \text { Dominio discursivo } \\
\text { - } & \text { Dominio textual } \\
\text { - } & \text { Convenciones de legibilidades }\end{array}$ & No se informan \\
\hline
\end{tabular}

Fuente: Elaborado por los autores

La limitación principal al analizar este tipo de informes es que están enfocados en contenidos específicos disciplinares y no contemplan una evaluación de competencias complejas donde se integren estas áreas de contenidos como, por ejemplo, la argumentación, y resolución de problemas en relación a la acción del ser humano en la deforestación y cambio climático o en acciones responsables que permitan cuidar nuestro planeta.

Las pruebas construidas desde una visión adulto céntrica invisibilizan las reflexiones y conocimientos que niñas y niños pueden tener sobre los distintos procesos que conforman su construcción identitaria y multidimensional. Se insiste en una visión de estancos vacíos y en una evaluación escindida de quienes aprenden, de los objetos que los configuran y de las estructuras de mundo que dan sentido a sus vivencias.

\section{PISA 2018 (OCDE, 2020)}

El Informe PISA Políticas efectivas, Escuelas exitosas (2018, Vol. I), que se reporta en OCDE (2020), se estructura en nueve capítulos: Cómo examina PISA las políticas eficaces y las escuelas exitosas; Cómo progresan los estudiantes en la educación; Clasificación y selección de estudiantes entre y dentro de las escuelas; Docentes y personal de apoyo; Recursos materiales 
disponibles en la escuela; Tiempo de aprendizaje durante y después del horario escolar; Escuelas privadas y elección de escuelas; Valoraciones, evaluaciones y cómo se utilizan; y, Las preguntas que los responsables de las políticas deberían plantearse.

Una de las limitaciones de este tipo de estudios es que se centra en el rendimiento y desempeño académico del estudiantado y en la interpretación de cifras que, en ocasiones, no responden a los diversos contextos invisibilizados en este tipo de análisis. Un ejemplo de ello es la sobre escolarización y la priorización respecto a un nuevo sistema de financiamiento de la Educación Parvularia en Chile que no cuenta con el apoyo del profesorado de Educación Parvularia, quienes han criticado el proyecto de ley y han alzado la voz para advertir las problemáticas que esto produciría en este importante nivel educativo.

El Informe declara que "la investigación ha identificado factores relacionados con los maestros que tienen un impacto medible en el rendimiento académico de los estudiantes o en el bienestar social y emocional de los estudiantes" (p. 88), sin embargo, en la lectura del mismo no se evidencian cuáles son esos factores relacionados y cómo se establece el impacto medible entre el rendimiento académico y el bienestar social y emocional del estudiantado.

Atendiendo a las limitaciones referidas en esta sección consideramos que es necesario incorporar una mirada crítica en las investigaciones que apunten a la mejora educativa, a los procesos de formación inicial y desarrollo profesional docente. Pensamos que en este nuevo milenio el desafío debería ser una mirada desde el ser humano complejo y multidimensional, ciudadano planetario consciente y responsable de su propia construcción histórica y del impacto que sus acciones generan en el planeta en el cual está inserto.

\section{Pensamiento complejo y complejidad vivencial: algunas ideas claves}

Morín (1990) plantea el pensamiento complejo como una postura filosófica que concibe al humano como un homo complexus en lugar de homo sapiens. Resalta que la animalidad y la humanidad constituyen juntas nuestra condición humana y que el concepto de hombre tiene un doble principio: uno biofísico y otro psico-socio-cultural. Ambos principios se remiten el uno al otro. Distingue como parte constituyente de lo humano a bucles inseparables que se codefinen entre sí: mente/cerebro/cultura, razón/afecto/impulso e individuo/sociedad/planeta (Morín, 1999). 
La complejidad es "un tejido de eventos, acciones, interacciones, retroacciones, determinaciones, azares, que constituyen nuestro mundo fenoménico (...) se presenta [la complejidad] con los rasgos inquietantes de lo enredado, de lo inextricable, del desorden, la ambigüedad, la incertidumbre" (p. 32). La complejidad es lo entretejido, un todo compuesto por hebras que respeta las texturas comunes, esas tesituras compartidas se forman para proyectarse más allá de sus partes (Morín, 1990).

Comprender el pensamiento complejo desde una mirada racionalista clásica, carece de sentido, puesto que se descentra el foco de lo característico y distintivo de lo humano en tanto su racionalidad y objetividad, desplazando la atención hacia una mirada interrelacionada, a través de una metáfora de entretejidos complejos.

Hay tres principios que ayudan a pensar la complejidad: el principio dialógico, que apunta a mantener la dualidad en el seno de la unidad, asociando términos a la vez complementarios y antagonistas; el principio de recursividad organizacional, una idea que rompe con el pensamiento lineal de causa/efecto, aquí todo lo que es producido se entreteje con aquello que lo ha producido en un ciclo en sí mismo auto-constitutivo, auto-organizador, y autoproductor; y, finalmente, el principio hologramático, que alude a la relación parte-todo, apelando a que no solamente la parte está en el todo, sino que el todo está en la parte. Con esto, se trasciende al reduccionismo que no ve más que las partes y al holismo que no ve más que el todo (Morín, 1990).

Con base en esta perspectiva, Ávila (2018a) acuña la noción de complejidad vivencial, entendida como aquella complejidad "que se constituye entrelazada a la experiencia del individuo al vivenciar lo humano, en la complejidad en que se encuentre inmerso" (p. 239). Dicha noción busca ir más allá de la complejidad que se instala con el pensamiento complejo, dado que al atender al humano en sí mismo como un ser complejo que vivencia, puede desprenderse que por estar el humano inmerso en un fenómeno complejo y tener éste la facultad de vivenciar emerge ese otro tipo de complejidad, la complejidad vivencial, que acontece como la experiencia humana que se da "justamente en el acto de vivenciar" (p. 239).

Una complejidad vivencial se da en el ser padre, ser madre. Algo inaccesible de manera externa por mucho que se busque comprender o empatizar la vivencialidad que en el momento de ser padre cobra forma, enactuando con ello un mundo que es propio e intransferible. Algo 
cercano desde el punto de vista del pensamiento complejo, sería aspirar a valorar y entender lo más empáticamente posible esa experiencia, no obstante, se sigue actuando desde fuera del cuadro del ser padre o madre, desde la no vivencialidad de esa maternidad, de esa paternidad. La complejidad vivencial sería eso: vivirla, sentirla, experienciarla, ser padre, ser madre. En el caso del aula de matemáticas: ser estudiante, ser maestro. Cada vivencia estudiantil y del maestro o de quien se involucre en el aula, se constituye como una complejidad vivencial en el sentido descrito, con interrelaciones, emociones, impulsos y comprensiones propias de lo que allí concurre y acontece (Ávila 2018b, p. 115).

Con esto, se tiene la intención de dar relevancia al estudio de las emociones desde un punto de vista vivencial, explorando trayectorias y flujos que las llevan a configurarse en la persona. Para ello distinguimos entre emoción por concurrencia y emoción por emergencia:

Una emoción emerge en una persona cuando al vivenciar ésta una situación que la involucra, cobran forma aspectos configurativos que hacen emerger a esa emoción, principalmente, a propósito de aquello que está aconteciendo en ese momento (...) Una emoción concurre en una persona cuando al vivenciar ésta una situación que la involucra la emoción también emerge, pero esta vez, más entrelazada a lo que porta desde su acervo experiencial. (Ávila, 2018a, p. 240)

Incorporar la faceta vivencial y la dimensión emocional en los estudios vinculados a la calidad o la mejora de los procesos de formación docente, consideramos que es necesario ya que con ello se presta atención a diferentes "nodos" involucrados en los espacios formativos docentes desde el punto de vista de los hablantes, que permiten distinguir y comprender con mayor profundidad aquello que se entrelaza y entra en juego en ese fenómeno complejo.

Con este planteamiento, se reportan a continuación complejidades vivenciales y emociones de estudiantes de pedagogía en matemáticas en su experiencia de práctica profesional y, también, de profesorado principiante de educación primaria.

\section{Emociones de estudiantes de pedagogía en matemáticas en práctica profesional}


Ávila, Moya, Díaz y Urbina (2018) exploran emociones del estudiantado de pedagogía en matemáticas en los momentos en que éstos vivencian sus espacios de práctica profesional. Desde una mirada compleja, buscan comprender tramas implícitas que configuran lo vivido por los sujetos en sus procesos de práctica profesional. Con un estudio de caso, se da cuenta de itinerarios de dos estudiantes que llevaron semanalmente un diario de reflexión acerca de sus experiencias en sus espacios de práctica, triangulando la información con entrevista en profundidad y notas de campo por parte de los investigadores. En este artículo, se da cuenta de resultados obtenidos a partir de las narrativas en el diario de reflexión de ambos casos (estudiante practicante $\mathrm{C} 1$ y $\mathrm{C} 2$ ).

Al enfrentarse a situaciones de enseñanza de la disciplina, ambos estudiantes evidencian emociones como la confianza, aceptación, interés, miedo e inseguridad, las cuales emergen junto a fenómenos que se suscitan en su práctica profesional.

El profesor me recomendó partir desde funciones hacia lo último que vimos. Al momento de partir tenía en mente ver cosas que salían en la prueba para tratar de ayudar a los alumnos, así que empecé con lo más simple, sin embargo, los alumnos desviaron la clase haciéndome preguntas para yo responderle las dudas que tenían, lo que me gustó más ya que mostró un mayor interés por parte de ellos. Me gustó mucho la clase porque aclaramos muchas dudas y reforzamos otros contenidos que aparecían en la prueba. [Diario de Reflexión, Estudiante C1]

En esta textualidad C1 manifiesta confianza, sorpresa y alegría frente al manejo de una situación de enseñanza centrada en un principio en él, pero que luego se desliza hacia las inquietudes e intereses de sus estudiantes, respecto de las dudas que éstos presentan. El interés demostrado por ellos fortalece su confianza y va favoreciendo la percepción de sí mismo frente a la docencia. La confianza aparece como emoción concurrente en el sentido que forman parte de la impronta con que se plantea previo a la clase, a su vez, al interactuar con los estudiantes a través de dar cabida al levantamiento de preguntas por parte de éstos, la sorpresa y la alegría confluyen como emociones por emergencia ya que entran a la escena vivencial de $\mathrm{C} 1$ a propósito de esas preguntas estudiantiles que describe que surgen en el momento en que él está llevando explicativamente la clase, lo que provoca incluso que dicha acción de enseñanza se amplíe al reforzamiento de otros contenidos, no considerados en su planificación inicial.

En otro momento, se analiza cómo emergen en este estudiante practicante emociones distintas a las observadas anteriormente, a partir de la siguiente textualidad: 
Hay ciertos aspectos matemáticos que me preocupan un poco, donde me doy cuenta que hay contenidos que manejo, sin embargo, no en la profundidad que se necesita, ya que en un momento unos estudiantes tenían dudas y les respondí, pero no de la mejor manera, lo que llevó a generar un error, del que corregí más adelante cuando aclaraba el mismo aspecto con otros estudiantes. Esas cosas me hacen perder un poco la confianza, pero obvio son cosas que uno debe aprender y que iré adquiriendo en el proceso. Ahora el profesor cuando me pregunta en la clase yo contesto con más seguridad y ya no me pongo tan nervioso. Creo que se debe a la misma confianza que ya hay en el ambiente. [Diario de Reflexión, Estudiante C1]

En este caso, C1 manifiesta sus miedos, inseguridades y frustración frente al dominio del contenido disciplinar. Sin embargo, reflexiona críticamente frente a su accionar lo que va generando en él nuevos aprendizajes desde el "ser profesor". Por dipolo contrario, se aprecia además la presencia de inseguridad y nerviosismo, al inicio de su proceso de práctica, cuando el profesor le preguntaba en clases, en tanto que en el momento temporal en el que efectúa la narrativa que se ilustra, se observa que aquello ya ocurre con menor intensidad. Esas vivencias previas en su contexto de práctica se entrelazan a este momento presente, en una sensación de alivio, de haber avanzado en esa cualidad. Hay un devenir continuo en el tiempo, de reflexión y auto mirada, imbricada a su emocionalidad.

De su discurso se desprende que esas emociones de seguridad y, ahora, mayor tranquilidad entran a escena debido al ambiente de confianza que ya se genera en el aula. Se elucida aquí que lo que muchas veces se arenga de manera externa al aula con relación a que "teniendo dominio del contenido, autoridad y una buena disciplina en el curso" empodera al futuro docente y le porta de seguridad, no siempre se cumple en esos términos, pues aquí, la seguridad y la tranquilidad confluyen principalmente por el ambiente de confianza en el aula, no por la implantación de una docencia autoritaria con base en el orden y la disciplina.

El estudiante en práctica C2 evidencia en sus narrativas la necesidad de valoración y, con esa impronta personal, la emoción de la aceptación por parte de su profesora guía se configura como una emoción que concurre con él en su contexto de práctica profesional:

La profesora me llamó la atención sobre esto, en específico en los tiempos que estaba demorando en terminar ejercicios. Conociendo muy bien a mi profesora guía, agradezco el tener a alguien que siempre va de frente, y me dice sin pudor mis fortalezas y debilidades. Sus consejos siempre los voy anotando en algunos rincones de mis libretas para luego reflexionar sobre eso. Ya en las clases siguientes (en "forma") fui más detallista con los tiempos, informando los minutos que tendrían para 
realizar ejercicios para luego revisar en conjunto. La profesora nota estos cambios y me hace saber que todo va bien. [Diario de Reflexión, Estudiante C2]

En otra narrativa, se observa que no tiene cuestionamientos hacia las estrategias que le indican para la apropiación del conocimiento matemático por parte de sus estudiantes, obedece y se centra en el "pasar contenidos":

En la clase de esta semana se plantea en la pizarra el objetivo de conocer el cuadrado y cubo de binomio. Con el pasar del tiempo la profesora me plantea solo pasar el cuadrado de binomio, y el cubo de binomio pasarlo al final de la unidad. En conclusión, me enfoqué solo en ese contenido y así en la próxima, para luego pasar a suma por su diferencia y binomio con factor común. [Diario de reflexión, Estudiante C2]

No existe un cuestionamiento didáctico hacia el docente o sus estrategias, tampoco hacia sí mismo y las planificaciones realizadas ante el no logro de los propósitos de la clase, se distingue sorpresa o conflicto, con relación a lo que acontece en el aula y lo que le habían infundido en la Universidad:

Desde la universidad me han enseñado que las fórmulas no se deben plantear porque sí, sino que se deben demostrar. Así que he decidido plantear una demostración por descomposición geométrica de cada uno de los productos notables. Luego de hacer la demostración del cuadrado de binomio cuando es una diferencia, me doy cuenta de lo complicado que lo encontraron los estudiantes del Primero medio $\mathrm{C}$ (curso de buen rendimiento). Lo hablo con la profesora y me dice que no lo demuestre en el Primero A. Le respondo que está bien, pero la demostración de los otros productos notables los haré. Es así como demostré cada uno de los productos notables, pero solo pidiendo la atención de los estudiantes al televisor, sin que anotaran nada más que la fórmula. [Diario de Reflexión, Estudiante C2]

Se observa un posicionamiento comprensivo de los procesos de enseñanza y aprendizaje por parte del estudiante desde el profesor expositor que dona conocimientos, no hay una visión de cambio con centro en el aprendizaje del estudiante, sino que está focalizada en el yo quiero transmitir un conocimiento, no en que los estudiantes descubran por ellos mismos o construyan.

La confianza y la seguridad se aprecian también en este estudiante, pero confluyen en el aula de manera distinta a como confluyen para $\mathrm{C} 1$. Como se ilustró anteriormente, para el caso de $\mathrm{C} 1$ dichas emociones emergen fruto de una interrelación más entrelazada y compartida en el aula; para C2, aun habiendo interrelación, la confianza y la seguridad se van instalando en él más bien por una relación asimétrica de la que se impregna gradualmente, con matices de cercanía, en su actuar y sentir: 
El tener más cursos, distintos contextos, me ha formado un poco más de carácter frente al desorden, frente a la falta de respeto del estudiante (...) Si me doy cuenta que no hay un buen ambiente para seguir con la clase, me detengo, converso con ellos, me doy ese tiempo y luego sigo (...) por más joven y simpático que se vea el profesor, deben entender que el profesor es un amigo, pero a la vez la autoridad en la sala (...) Estos contextos en los que me veo enfrentado me fortalecen, en lo que es mi seguridad y manejo de grupo. Los acuerdos y conversaciones que se tienen con los alumnos generan un ambiente de confianza y cercanía. [Diario de Reflexión Estudiante C2]

Aquí, las emociones de confianza y cercanía van emergiendo de acuerdo al contexto e interrelación con sus estudiantes, por una parte, como emoción emergente en tanto que entra a escena propiciada por esos momentos interactivos a que alude, pero, a su vez, puede interpretarse que también fungen como emociones concurrentes, ya que se van configurando junto con su necesidad de validación, en este caso, a partir del manejo de grupo. Es decir, la seguridad y confianza, en este caso emergen a partir de una lógica del dominio de una de las partes, más que de lo que emerge a partir de la relación entre las partes.

En el sentido descrito entonces, se vislumbra una confianza diferente a aquella que se analizaba en el caso del estudiante $\mathrm{C} 1$, en donde la confianza en ese caso "se reconocía en el ambiente" (ver cuadro 3).

Cuadro 3

Entretejido de emociones y complejidad vivencial para la confianza en la Gestión del aula

\begin{tabular}{|l|l|l|l|l|}
\hline \multirow{2}{*}{$\begin{array}{l}\text { Confianza, } \\
\text { seguridad }\end{array}$} & \multicolumn{2}{|c|}{ C1 } & \multicolumn{2}{c|}{ C2 } \\
\cline { 2 - 5 } & $\begin{array}{l}\text { Alegría } \\
\text { sorpresa }\end{array}$ & $\begin{array}{l}\text { Relación } \\
\text { simétrica (actuar } \\
\text { desde ra } \\
\text { interacción, } \\
\text { clima relacional) }\end{array}$ & Validación & $\begin{array}{l}\text { Relación asimétrica } \\
\text { (automirada como una } \\
\text { autoridad) }\end{array}$ \\
\hline
\end{tabular}

Fuente: Elaborado por los autores

En el estudio se reconocen además emociones vinculadas a otros aspectos del contexto de práctica profesional, para ambos casos, $\mathrm{C} 1$ y C2, las emociones que emergen a propósito de la relación con sus estudiantes les marca notoriamente. Un aspecto relevante es la satisfacción y alegría que les provoca el ayudar a sus estudiantes, se sienten útiles y que están aportando. Al mismo tiempo, en ambos, la emoción de la preocupación confluye ante la inminente pérdida de credibilidad o desaprobación por parte de sus estudiantes cuando por alguna circunstancia queda en evidencia que ellos no saben algo. 
Para $\mathrm{C} 1$, el patio y la relación persona a persona con sus estudiantes provoca satisfacción, una emoción que en ese contexto informal del patio emerge más por concurrencia ya que tiene estrecha relación con su planteamiento de vida, de un querer tener cercanía con sus estudiantes a fin de hacer del colegio algo más familiar y humano. La sala de profesores para C1 forma parte de un cuadro emocional más bien de lejanía y frialdad.

Otro aspecto propio a la complejidad vivencial que se pudo distinguir para $\mathrm{C} 1$ es que la cercanía con sus estudiantes en sus espacios de práctica -que le generan emociones de alegría y satisfacción- por una parte, lo hacen sentirse cómodo y con mayor confianza a medida que transcurre el tiempo, pero acercándose el momento de tener que efectuar las clases en ese mismo curso, comienzan a configurarse y emerger la ansiedad y el miedo. Se distingue así, un entretejido de emociones con diferente cariz asociadas a la gestión del aula y que confluyen (cobran forma) en conexión con una misma faceta de su complejidad vivencial, vinculada en este caso, a su buena relación y cercanía con sus estudiantes.

\section{Emociones de profesorado principiante de educación primaria en su inserción profesional}

En palabras de Cabello, Ruíz-Aranda \& Fernández-Berrocal (2010) “Ser profesor, como ser persona, se ha convertido en el siglo XXI en una tarea más difícil de lo que podríamos haber imaginado cada uno de nosotros cuando comenzamos a formarnos como docentes" (p. 47). En este sentido, consideramos que explorar las emociones en el contexto de la complejidad vivencial del profesorado implicado en sus procesos de inserción profesional, coadyuva a comprender con mayor profundidad lo que allí emerge para aportar con elementos de realidad a tener en cuenta para una mejora de las políticas de formación docente.

Vélaz (2009) plantea que el profesorado principiante:

Se incorpora con una gran inseguridad -que generalmente se afronta y supera en soledad-sobre cómo responder al día a día en el aula y en la escuela, sin haber tenido en la mayoría de los casos la oportunidad de aprender realmente los saberes más prácticos del oficio, ni cómo movilizar los saberes teóricos y las metodologías aprendidas, y cómo generar conocimiento a partir de la reflexión sobre la práctica. (p. 210) 
Desde nuestra perspectiva de la complejidad, vemos importante agregar que el profesorado principiante, como ser complejo y vivencial, inevitablemente se inserta en ese nuevo mundo con todas sus características biográficas e identitarias, con todos sus anhelos, ilusiones, convicciones, miedos y esperanzas. Cabe preguntarse entonces cómo esa inserción gatilla sus afectos y emociones, necesidad de aceptación por parte de sus colegas y estudiantes, necesidad de sentirse querido y considerado por la comunidad en la que se incorpora. Por una parte, debe aprender a aplicar los conocimientos aprendidos en su formación inicial, pero, a la vez, debe impregnarse de los nuevos conocimientos y prácticas que deberá aprender sobre la cultura de la institución, manejo administrativo, laboral, comunicativo y relacional.

En el contexto de una investigación en curso se exploran tensiones emocionales que confluyen en la inserción profesional de profesorado principiante de educación primaria. La información se recolecta por medio de narrativas biográficas, entrevistas en profundidad y grupos de discusión. En este artículo, se da cuenta de resultados preliminares a partir de las narrativas biográficas de dos profesores (P1 y P2) considerados para el estudio.

Se reconocen emociones que emergen y concurren como la anticipación, preocupación, admiración, desilusión y decepción, que surgen entretejidas a la complejidad vivencial que cobra forma en los espacios de inserción profesional, y que tiene que ver, entre otros aspectos, con cómo sienten la profesión docente, lo que desean para sus estudiantes, su visión acerca del gremio y las políticas educativas.

Lo primero que se me viene a la mente cuando se nos da la posibilidad de narrar la experiencia profesional, se relaciona con el lograr que nuestros alumnos obtengan los mejores resultados en el colegio, entendiendo como mejores resultados no solamente lo relacionado a sus notas sino lo concerniente principalmente a su formación como ser humano. Es importante estar conscientes que uno no sólo los forma en el área que se enseña, también los forma en el ámbito valórico, es el eslabón para que el niño ingrese en una vida de adulto (...) Por otro lado inquietantemente me pregunto qué tan motivante son cada clase que se ha preparado. Soy lo suficientemente estimulante o sólo me quedo en la pasividad. Existe algún grado de apatía con algunos de mis alumnos. Existe parcialidad en mis enseñanzas con respecto a determinados estudiantes. Son verdaderamente fructíferas mis clases. Encaminan hacia los objetivos que se han determinado y concuerdan con los pasos antes planificados (...) En mi proceso de ejecución y la impartición de la enseñanza veo que existe una gran cantidad de problemas de aprendizajes y que debemos buscar respuestas (...) muchas veces debo 
estructurar y desestructurar para pulir detalles sin que los estudiantes se den cuenta. [Narrativa biográfica, Prof. principiante P1]

Desde mi punto de vista, la clave está en que la vida y la profesión son inseparables, sobre todo si es un camino que no te impusieron, es el que uno eligió, sistemáticamente hablando, el proceso de enseñar se dirige estrictamente a que el docente debe ser un ente integral para los estudiantes, probablemente así, la formación sea más adecuada técnicamente y emocionalmente para los estudiantes. [Narrativa biográfica, Prof. principiante P2]

En esta complejidad vivencial, en P1, confluyen emociones como la anticipación y la preocupación, que se derivan principalmente por el sentirse comprometido con los aprendizajes reales de sus estudiantes, más que desde el punto de vista del rendimiento ("sus notas"), su compromiso se liga con un fuerte sentido de responsabilidad por los problemas de aprendizajes y desarrollo integral de sus estudiantes, como seres humanos. Además, es una tarea que se vivencia como una labor que se realiza con entrega, convicción y de manera solitaria, en particular, cuando detecta problemas de aprendizaje y cuando quiere ir más allá, en su afán de formarlos como seres humanos. Un motor sustancial para este docente es el amor y compromiso con sus estudiantes, se debe completamente a ellos.

Para P2, el sentirse comprometido, aparece estrechamente vinculado a una convicción de vida con respecto a su profesión ("la vida y la profesión son inseparables") y a su convencimiento con relación al rol docente ("debe ser un ente integral [el docente] para los estudiantes"), ambos aspectos forman parte de su complejidad vivencial en el aula.

El sentirse comprometido se imbrica a la vez con emociones como la desilusión, la decepción o la rabia, por la ausencia de políticas educativas que se hagan cargo de velar por este fuerte aspecto de entrega, que de un modo u otro incide en la formación de la ciudadanía (para P1) o, también, porque no se ve en otros colegas un compromiso profesional (para P2):

Desde mi perspectiva las primeras y más importantes políticas educacionales deben ir dirigidas a encauzar a aquellos estudiantes de pedagogía de todo el país para permitirles decidir con conocimiento si es la educación en aula el camino que desean tomar para su futuro, aconsejarlos sobre la importancia que tiene un docente en la vida de un niño, la capacidad que posee de convertirlo en una buena persona guiándolo de la forma correcta. Me parece pertinente que este sea un tema de discusión en nuestras escuelas, en nuestras casas de estudios, en nuestros hogares. [Narrativa biográfica, Prof. principiante P1] 
Muchos de nuestros compañeros de trabajo nunca quisieron ser docentes. Llegaron por diversas situaciones de su vida, muchos ingenieros frustrados nos acompañan en las clases de matemáticas, otros tantos escritores en lenguaje y la lista continúa. Por sobre cualquier dificultad que podamos encontrar en nuestra labor, me parece que es ésta la más terrible. Una persona que no se desarrolla íntegramente en su profesión u oficio compromete absolutamente todos los esfuerzos por revertir los grandes errores que se han cometido en materia de educación. [Narrativa biográfica, Prof. principiante P2]

En una situación de diversidad de realidades que pueden darse en el proceso de inserción laboral, al trabajar en diferentes establecimientos, también confluyen variadas emociones:

En mis primeros años de docencia he conocido muchas realidades, algunas muy hermosas sacadas de libros ideales con buenas prácticas directivas, administrativas y docentes, en otras muy adversas pero con profesionales que luchan a diario, sabiendo que son batallas perdidas de antemano con alumnos, si se les puede llamar así, son ejemplos de entrega y vocación que me han dejado en más de una oportunidad sin palabras y en muchos otros lugares en donde existiendo la posibilidad de tener buenas prácticas no las hay, no porque no haya recursos ni infraestructura, sino porque no hay voluntad de tenerla, generando un círculo vicioso. [Narrativa biográfica, Prof. principiante P2]

En esta narrativa se distinguen emociones que van desde la alegría por el reconocimiento de buenas prácticas de diferentes actores del contexto escolar, admiración y sorpresa, por el actuar docente de sus pares en situaciones de adversidad, hasta la decepción por el desaprovechamiento en los centros educativos de los recursos que estos puedan poseer.

En P1, se reconocen además emociones vinculadas a su profesión y a sus estudiantes:

Muchas veces los alumnos matan la ilusión de un profesor, con su mala conducta, rebeldía y faltas, pero es porque en algún punto determinado un profesor mató sus ilusiones y entregó pautas equivocadas de comportamiento ...durante mis horas de trabajo trato de eliminar las continuas frases antes mencionadas y lleno mi mente solo con la frase que tengo en mi escritorio......recuerda son solo niños, son solo niños que esperan que desarrollemos la mejor de las labores, que no votan, por lo que no eligen a quienes decidirán su futuro escolar, no saben de políticas educacionales, están ahí solo esperando que los eduquen y que los quieran. [Narrativa biográfica, Prof. principiante P1]

Se observa que busca comprender a sus estudiantes, no los cataloga ni demoniza por sus acciones conductuales, de rebeldía o falta como algo intrínseco que pueda surgir a partir de ellos, sino que lo relaciona con una posible acción docente pasada que influyó en ellos. Pese al 
comportamiento de sus estudiantes o a las críticas del medio externo hacia la profesión docente, más allá de las políticas educacionales, se observa que lo que impera en su labor docente son emociones como el interés, la confianza y el amor por su profesión y sus estudiantes, con base en una actitud empática hacia ellos.

También se observan experiencias de inserción en donde los primeros días que vive el docente principiante le invade el miedo y la inseguridad respecto a aquello que no le fue enseñado; y los roces con los otros profesores o miembros de la comunidad educativa. El conflicto que puede ser determinante a la hora de seguir o abandonar su labor profesional.

Fue una inserción algo pobre, poco guiada, debí estar a la siga de los coordinadores para que me explicaran cómo funcionaba el colegio. Nadie te ayudaba, todos te hacen el quite y para lo único que te hablan es para que vayas a cuidarles los cursos. Un momento significativo fue cuando para el día de la solidaridad mis alumnos me fueron a buscar para que compartiera con ellos. Me llevo bien con los niños porque me da rabia que muchos colegas ni preparan sus clases, es la ley del mínimo esfuerzo y eso perjudica a los cabros. [Narrativa biográfica, Prof. principiante $\mathrm{P} 2]$

Narrativas como las aquí ilustradas retratan situaciones complejas que no se visibilizan en los informes asociados a las políticas educativas. No hay que olvidar que el profesorado actual proviene prácticamente en su totalidad desde un sistema escolar y de formación profesional anclado en el siglo pasado y deben responder a las necesidades de nuevas generaciones provenientes de este nuevo siglo, un siglo que se debate entre los paradigmas arcaicos que van muriendo y los nuevos paradigmas que van surgiendo o surgirán.

\section{A modo de cierre}

Las tensiones entre las políticas educativas que se plasman en los informes analizados en este artículo, la búsqueda de resultados que apunten hacia la calidad de los procesos de enseñanza en la Escuela y el desencanto del profesorado en ejercicio con más años de experiencia, caracteriza fuertemente a los contextos educativos. En este escenario son múltiples las causales de la crisis educativa donde los programas de formación docente siguen sin incorporar el hecho que estamos en un nuevo siglo donde las tecnologías avanzan a un nivel vertiginoso dejando día a día obsoleto la transmisión de contenidos y donde las y los jóvenes a quienes se está formando tienen otros códigos para desenvolverse en este mundo cambiante. 
Las políticas educativas deberían coadyuvar y favorecer a que los establecimientos educativos dejen de estar estructurados con características de las escuelas de siglos pasados, e incorporen las necesidades de las nuevas generaciones a educar y las resignificaciones que se están desarrollando con respecto a la naturaleza humana en este nuevo milenio.

Ante un escenario de cambio de cosmovisión de mundo, considerando las características de las nuevas generaciones, se ve urgente el plantearse una agenda que permita redefinir las estructuras educativas, a fin de hacerlas más pertinentes y acordes a los modos de ser y de pensar en estos nuevos tiempos.

Consideramos que las palabras de Imbernón (2008), resultan ser muy actuales, ante el contexto y temática abordada en el presente artículo:

Si nos adentramos ya en la formación permanente, vemos que el conocimiento que se ha creado sobre ella en los últimos decenios nace en una época de cambios vertiginosos, donde todo lo que nace, se crea, se diseña, etc., empieza a ser obsoleto y caduco en el momento de surgir. Ello obliga a plantearse una reconceptualización constante, es decir, una reflexión de zonas intermedias de la práctica como son la singularidad, la incertidumbre y el conflicto de valores (Schön, 1992) y una indagación perenne sobre la formación del profesorado, ya sea inicial o permanente. Y es ahí donde aparecen problemas, ya que es más fácil instalarse en lo que bien o mal ha ido funcionando que arriesgarse a introducir cosas nuevas, aunque sean necesarias (Imbernón, 2008, p. 16).

Con relación a la aproximación utilizada para el estudio de las emociones desde la perspectiva de la complejidad vivencial y las ejemplicaciones vertidas en este artículo, cabe señalar que se busca diferenciarse de las aproximaciones cognitivas más usuales para el estudio de la emoción en la formación de profesorado, puesto que aquellas ponen el foco de atención principalmente en el análisis de la emoción ya cristalizada (una vez ocurrida) y en sus implicancias funcionales. La principal diferenciación con la perspectiva que aquí se propone radica en que en nuestro caso se busca visibilizar aquellos elementos que inciden en la configuración de una emoción desde el punto de vista experiencial de los sujetos, atendiendo al contexto y a lo que ellos vivencian (con una situación o con los actores involucrados en una 
situación) considerando aspectos como lo biográfico pero también lo que acontece en sus experiencias previas más próximas (inmediatas incluso) a la ocurrencia de sus emociones, reconociéndolas siempre en el contexto situacional y experiencial del sujeto que las vivencia. Con este posicionamiento epistemológico, se busca atender también a lo que las emociones van configurando en los sujetos desde un punto de vista vivencial.

En síntesis, la apuesta de estudio está en el desafío de prestar atención a los aspectos y elementos propios a la complejidad vivencial de los sujetos, que configuran a sus emociones y, a su vez, prestar atención a cómo sus emociones van configurando a aquellos elementos en esa complejidad vivencial.

\section{Referencias}

Ávila, J. (2018a). Emergencia y concurrencia de emociones en el proceso formativo del profesorado de matemáticas. Transformación. Revista electrónica científico pedagógica, 14 (2), 236-251. $\quad$ http://scielo.sld.cu/scielo.php? script=sci abstract\&pid=S207729552018000200009

Ávila, J. (2018b). Configuraciones emocionales en estudiantes de profesorado de matemáticas. Un estudio desde la perspectiva del pensamiento complejo (tesis doctoral). Universidad de Los Lagos, Santiago, Chile.

Ávila, J.; Moya, M.; Díaz, L. y Urbina, M. (2018). Caracterización de las emociones que concurren en estudiantes de pedagogía en matemáticas e informática educativa, en el contexto de su práctica profesional. Informe de avance, Proyecto de Investigación Fondo Semilla UCSH 2018. Santiago, Chile: UCSH.

Bächler, R., Meza, S., Mendoza, L. y Poblete, O. (2020). Evaluación de la Formación Emocional Inicial Docente en Chile. Revista de estudios y experiencias en educación, 19(39), 75-106. https://dx.doi.org/10.21703/rexe.20201939bachler5

Badia, A. (2014). Emociones y sentimientos del profesor en la enseñanza y la formación docente. En C. Monereo (Coord.). Enseñando a enseñar en la universidad (pp. 62-90). Barcelona:

Octaedro/ICE-UB. https://www.researchgate.net/publication/305493634_Emociones_y_sentimientos_del_prof esor_en_la ensenanza_y_la_formacion_docente

Cabello, R., Ruíz-Aranda, D. y Fernández-Berrocal, P. (2010). Docentes emocionalmente inteligentes. Revista Electrónica Interuniversitaria de Formación del Profesorado, 13(1), 41-49. https://www.redalyc.org/pdf/2170/217014922005.pdf

Casassus, J. (2015) La educación del ser emocional. Chile: Editorial Cuarto Propio. 
Casillas, M. y Ramírez, A. (2019). Cultura digital y cambio institucional de las universidades. Revista de la educación superior, 48(191), 97-111. http://www.scielo.org.mx/scielo.php?script=sci arttext\&pid=S0185-27602019000300097

CEPAL-UNESCO (2020). La educación en tiempos de la pandemia de COVID 19. https://repositorio.cepal.org/bitstream/handle/11362/45904/S2000510 es.pdf? sequence $=1 \&$ isAllowed $=\mathrm{y}$

Cózar-Gutiérrez, R., De Moya-Martínez, M., Hernández-Bravo, J. y Hernández-Bravo, J. (2016). Conocimiento y Uso de las Tecnologías de la Información y las Comunicaciones (TIC) según el Estilo de Aprendizaje de los Futuros Maestros. Formación universitaria, 9(6), 105-118. https://dx.doi.org/10.4067/S0718-50062016000600010

Day, C. (2011). Pasión por enseñar. La identidad personal y profesional del docente y sus valores. 3ra edición. Madrid: Narcea, S.A. de Ediciones.

Gómez-Chacón, I. M. (2010). Tendencias actuales en investigación en matemáticas y afecto. En M.M. Moreno, A. Estrada, J. Carrillo, \& T.A. Sierra, (Eds.), Investigación en Educación Matemática XIV (pp. 121-140). Lleida: SEIEM.

Hargreaves A. (2005) The Emotions of Teaching and Educational Change. In: Hargreaves A. (eds) Extending Educational Change. Springer, Dordrecht. https://doi.org/10.1007/1-40204453-4_14

Hargreaves, A. (2001). The emotional geographies of teachers' relations with colleagues. International Journal of Educational Research, 35(5), 503-527. https:/www.sciencedirect.com/science/article/abs/pii/S088303550200006X

Imbernón F. (2008). 10 ideas clave. La formación permanente del profesorado. Nuevas ideas para formar en la innovación y el cambio. Barcelona: Editorial Graó.

Morín, E. (1990). Introducción al pensamiento complejo. México: Editorial Gedisa.

Morín, E. (1999). Los siete saberes necesarios para la educación del futuro. Francia: UNESCO.

Najmanovich, D. (2001). El sujeto encarnado: Limites devenir e incompletud. O Sujeito Encarnado. Questões para pesquisa no/do cotidiano. Brasil: Editora Río de Janeiro.

OCDE/CERI (2009). La comprensión del cerebro. El nacimiento de una ciencia del aprendizaje. [Traducción] Chile: Ediciones UCSH. https://read.oecdilibrary.org/education/la-comprension-del-cerebro-el-nacimiento-de-una-ciencia-delaprendizaje_9789567947928-es\#page2

OCDE (2020). Resultados de PISA 2018 (Volumen V): Políticas efectivas, Escuelas exitosas, PISA, Publicaciones de la OCDE, París, https://www.oecd-ilibrary.org/education/pisa-2018results-volume-v ca768d40-en

OECD (2019). TALIS 2018 Results (Volume I): Teachers and School Leaders as Lifelong Learners, TALIS, OECD Publishing, Paris, https://doi.org/10.1787/1d0bc92a-en. 
Ordorika, I. (2020). Pandemia y educación superior. Revista de la educación superior, 49(194), 1-8. http://resu.anuies.mx/ojs/index.php/resu/issue/view/40

Pardo, A. (2018). Desafios de la gestión de aula. Ficha VALORAS. Disponible en Centro de Recursos VALORAS: www.valoras.uc.cl

SITEAL (2014). Informe sobre tendencias sociales y educativas en América Latina. Políticas TIC en los sistemas educativos de América Latina. http://archivo.siteal.iipe.unesco.org/informes/archivo

Tarabini, A. (2020). ¿Para qué sirve la escuela? Reflexiones sociológicas en tiempos de pandemia global. Revista de Sociología de la Educación-RASE, 13 (2), Especial, COVID19, 145-155. https://doi.org/10.7203/RASE.13.2.17135.

UNESCO (2015). Informe de resultados TERCE, tercer estudio regional comparativo y explicativo: logros de aprendizaje. Laboratorio Latinoamericano de Evaluación de la Calidad de la Educación. https://unesdoc.unesco.org/ark:/48223/pf0000243532

Varela, F. (1990). Conocer. Las ciencias cognitivas: tendencias y perspectivas. Cartografía de las ideas actuales. España: Gedisa.

Varela, F. (2000). Conversaciones con Francisco Varela [Entrevista por Eric Goles]. Obtenido en noviembre $12, \quad 2014 \quad$ de http://inalambrico.reuna.cl/fichas/entrevistas/francisco_varela.htm

Vélaz, C. (2009). Competencias del profesor-mentor para el acompañamiento al profesorado principiante. Revista de currículum y formación del profesorado, 13(1), 209-229. https://recyt.fecyt.es/index.php/profesorado/article/view/42165

Zembylas, M., Watkins, M., Becerra, M. y Aleu, M. (2019). Emociones y afectos en el mundo educativo. Propuesta Educativa 51. ISSN 1995-7785. Flacso: Argentina. http://propuestaeducativa.flacso.org.ar/wpcontent/uploads/2019/11/PropuestaEducativa51.pdf 